\title{
Ultrastructure of an Indigotin-producing Dome Mutant of Schizophyllum commune
}

\author{
By ROGER MARCHANT \\ School of Biological and Environmental Studies, New University of Ulster, \\ Coleraine, County Londonderry, Northern Ireland \\ MARJATTA RAUDASKOSKI \\ Department of Botany, University of Turku, Turku, Finland \\ AND YONAH SHNEYOUR \\ Genetics Unit, Faculty of Life Sciences, Tel-Aviv University, Tel-Aviv, Israel
}

(Received I7 May 1976)

\section{SUMMARY}

Electron microscopic observations of an indigotin-producing dome mutant of Schizophyllum commune Fr. have shown that large wall ingrowths occur within the hyphae. These ingrowths are coupled with morphological abnormalities produced by the dome mutation. The pigment indigotin appears to be produced by progressive condensation within vacuoles and to a lesser extent within the wall ingrowths. Cytochemical techniques have shown that the wall ingrowths are similar in structure to the hyphal walls. There was no evidence for the passage of condensed indigotin into the medium; the pigment granules found in the medium must therefore form outside the hyphae.

\section{INTRODUCTION}

Schizophyllum commune Fr. is one of the few basidiomycetes which has been used extensively for genetical studies. The knowledge of the genetical systems operating in this organism has been used as a basis for physiological and biochemical studies (Wessels \& Niederpruem, 1967). Ultrastructural studies have also been undertaken, often specifically coupled with genetical, physiological and biochemical investigations (Koltin \& Flexer, 1969; Raudaskoski, 1973; Wessels \& Marchant, 1974).

The purpose of our study was to establish the structural basis of two mutations contained in a single strain. The two mutations are those leading to the production of a blue, waterinsoluble pigment (indigotin) (Miles, Lund \& Raper, 1956) and to a non-spreading colonial morphology (dome) (Raper, Baxter \& Ellingboe, 1960). The production of the blue pigment was first recorded by Papazian (1950) and it was identified by Miles et al. (1956). Indigotin is produced by many strains of $S$. commune (Raper \& Miles, 1958) and these have been used to study the conditions for production of indigotin (Swack \& Miles, 1960), the relationship between indigotin and indirubin production (Epstein \& Miles, I966) and the relationship between indigotin and indole-3-acetic acid production (Epstein \& Miles, 1967).

Papazian (1950) first reported the indigotin mutation in association with a morphological mutant character termed streak. In this study the pigment production appears to be closely linked with the morphological dome mutation, which is known to map close to streak (Raper et al., 1960). Although non-dome strains can be induced to produce small amounts of the pigment, the dome strains always produce large quantities under suitable conditions. 
This investigation sought to examine both the basis for the dome mutation morphology and the mechanism by which the pigment indigotin accumulates in the hyphae.

\section{METHODS}

Organism. The strains of Schizophyllum commune Fr. used were: strain 699, a wild-type homokaryon; and strain 45, which has closely linked dome and indigotin mutations.

The organisms were grown on small strips of cellophane membrane laid over complete medium (Snider \& Raper, I958). For phase-contrast microscopy, small pieces of cellophane membrane, with attached hyphae, were removed from the margin of the colony and mounted in $14 \%(\mathrm{w} / \mathrm{v})$ gelatin. The preparations were viewed through a Reichert Zetopan microscope and photographed, using microflash equipment, on Kodak Tri X film.

Electron microscopy. Pieces of cellophane membrane, with adhering hyphae, were removed from the margin of the colony and fixed for $4 \mathrm{~h}$ in $\mathrm{I} .5 \%(\mathrm{v} / \mathrm{v})$ glutaraldehyde in $0 . \mathrm{I} \mathrm{M}-$ phosphate buffer $(\mathrm{pH} 7 \cdot 2)$. After washing, the material was post-fixed for $2 \mathrm{~h}$ in phosphatebuffered ( $\mathrm{pH} 7 \cdot 2$ ) osmium tetroxide, dehydrated in an ethanol series, and embedded in Epon. Sections were cut with a diamond knife on a Huxley Ultramicrotome Mark II and viewed in an AEI EM 6 G electron microscope.

Cytochemistry. Lipid was removed from sections with sodium methoxide using a method modified from Eurenius \& Jarskär (1970). The sodium methoxide solution was prepared by dissolving $2.5 \mathrm{~g}$ powdered sodium methoxide in a mixture of $25 \mathrm{ml}$ methanol plus $25 \mathrm{ml}$ benzene. Sections were mounted on uncoated, 200 mesh, stainless-steel grids and extracted for 3 to $7 \mathrm{~min}$ with the sodium methoxide solution diluted $\mathrm{I}: 3$ with the methanol/ benzene solvent. After extraction, the sections were washed for 2 min each in methanol/ benzene $(\mathrm{I}: \mathrm{I}, \mathrm{v} / \mathrm{v})$, methanol, and twice in distilled water. Grids were stained for $\mathrm{I} 0 \mathrm{~min}$ in saturated uranyl acetate in $70 \%(\mathrm{v} / \mathrm{v})$ methanol followed by $30 \mathrm{~s}$ in lead citrate.

Carbohydrate was localized in sections by the method of Thiéry (1967). Sections were mounted on uncoated, 200 mesh, stainless-steel grids and treated as follows: (i) I \% (w/v) aqueous periodic acid for $20 \mathrm{~min}$; (ii) four $4 \mathrm{~min}$ washes in water; (iii) I \% (w/v) thiosemicarbazide in $10 \%(\mathrm{v} / \mathrm{v})$ acetic acid for $40 \mathrm{~min}$; (iv) three $5 \mathrm{~min}$ washes in $10 \%$ acetic acid; (v) washed in a graded series from $10 \%$ acetic acid to water; and (vi) exposed to osmium tetroxide vapour at $60{ }^{\circ} \mathrm{C}$ for 40 to $60 \mathrm{~min}$. The sections were examined without further staining.

\section{RESULTS AND DISCUSSION}

\section{Light microscopy}

The dome mutant showed visible pigment production after 3 to 4 days incubation at $30^{\circ} \mathrm{C}$. At this stage pigment granules were present in the medium as well as within the hyphae. This poses the problem of whether the pigment within the hyphae is a preliminary stage prior to excretion into the medium. The morphology of the dome mutant hyphae (Fig. 2) is totally dissimilar to that of the wild-type homokaryon hyphae of strain 699 (Fig. I). The hyphae of the mutant are distorted in outline, with branching occurring close to the hyphal apex. Individual hyphae of the mutant showed periods of apparently normal extension growth followed by a period of abnormal morphology. Few internal structural differences were seen associated with the morphological abnormalities, although opaque particles, presumably pigment, were clearly visible in the hyphae of the mutant strain. 

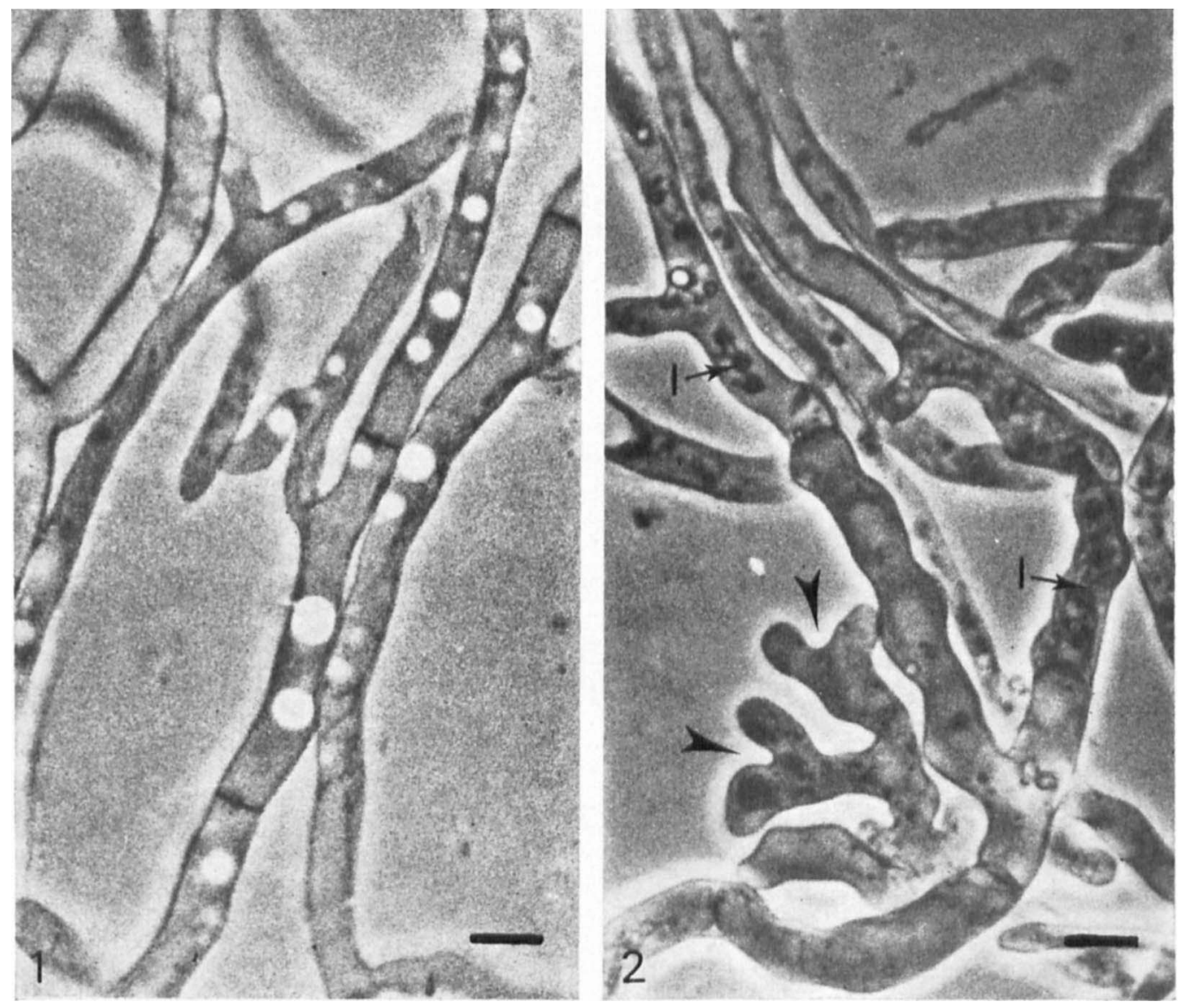

Bar markers represent $5 \mu \mathrm{m}$.

Fig. I. Phase-contrast micrograph of hyphae of the wild-type strain 699.

Fig. 2. Phase-contrast micrograph of hyphae of the indigotin-producing dome mutant strain 45 . Opaque particles in the hyphae (I) are probably indigotin granules. The abnormal morphology is exemplified by the distorted hyphal outlines and the apical branching (arrowed).

\section{Electron microscopy}

The most obvious features visible in sections of the mutant are shown in Figs 3 and 4. The outlines of the hyphae are distorted and large structures, apparently ingrowths of the walls, are present in many hyphae. Areas of electron-opaque material are visible sequestered within these ingrowths and within the cytoplasm. Large membrane complexes are a prominent feature of many hyphae (Fig. 6) and seem to enclose electron-opaque material. Sections treated by the Thiéry technique show (Fig. 5) that the large electron-transparent structures are wall proliferations. Furthermore the complex membrane structures also react strongly (Fig. 7), indicating that they are heavily contaminated with polysaccharide material.

The sequence of events that we propose is that membrane complexes, derived from the plasmalemma, become localized sites of wall polysaccharide accumulation. Small amounts of indigotin may be formed or included within the complex. As the process proceeds, the membrane complexes lose their original shape, due to the deposition of wall material, and 

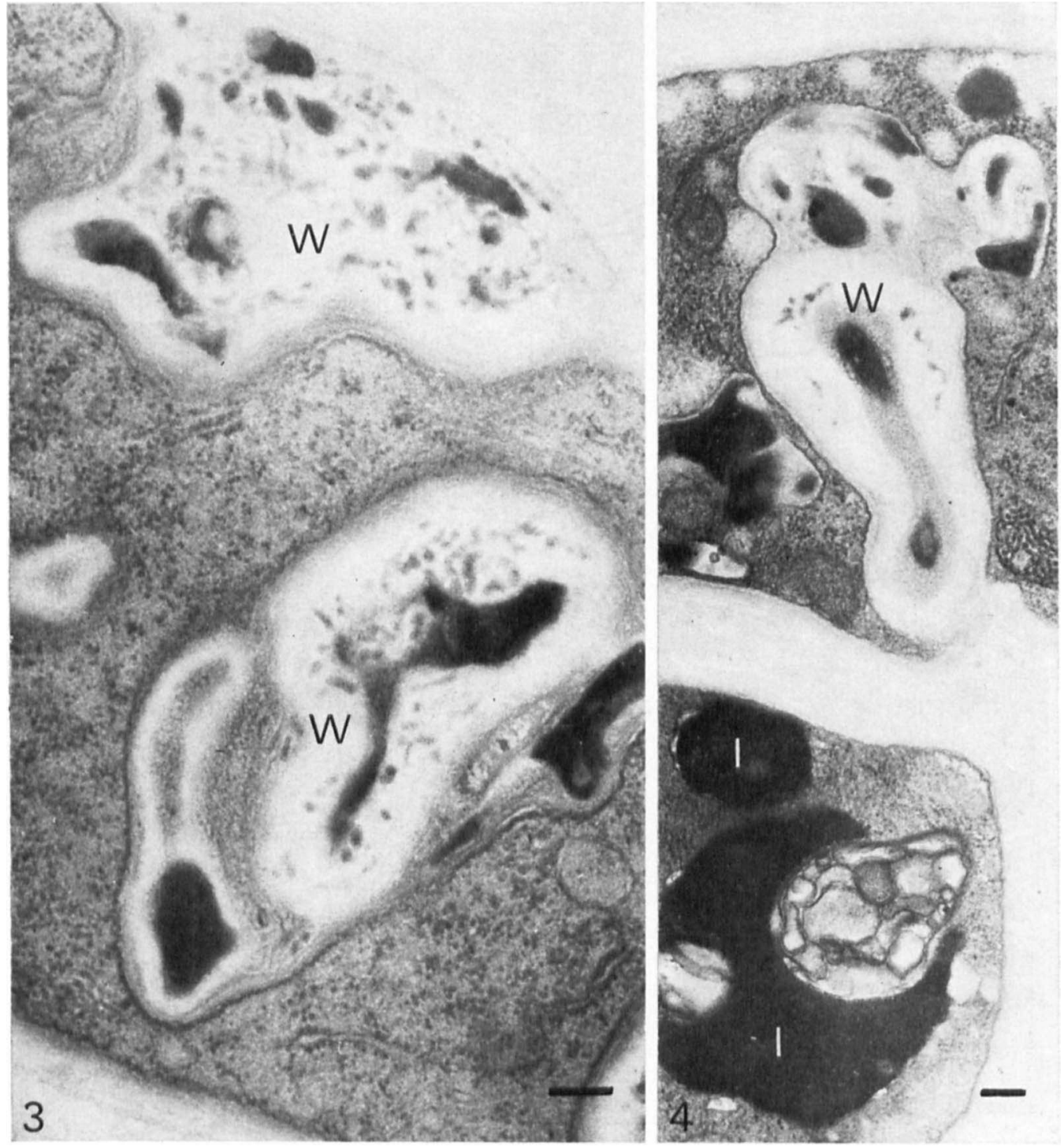

Bar markers represent $0.2 \mu \mathrm{m}$.

Fig. 3. Section through a hypha of the dome mutant, showing large wall ingrowths (W) containing electron-opaque material.

Fig. 4. Section through hyphae of the dome mutant, showing wall ingrowths (W) and electronopaque inclusions (I) in the cytoplasm.

indigotin may become trapped within the structure. This type of development, involving the sequestering of a membrane structure into a wall structure, has been discussed by Marchant \& Moore (1973). The plasmalemma does not react to the Thiéry technique (Fig. 7) and this lends further weight to the argument that these membrane proliferations are not merely fixation artefacts. Wall ingrowths of the type observed in this mutant have not been reported for wild-type strains of Schizophyllum commune (Raudaskoski, 1973), and are therefore certainly a manifestation of the dome mutation. The development of 

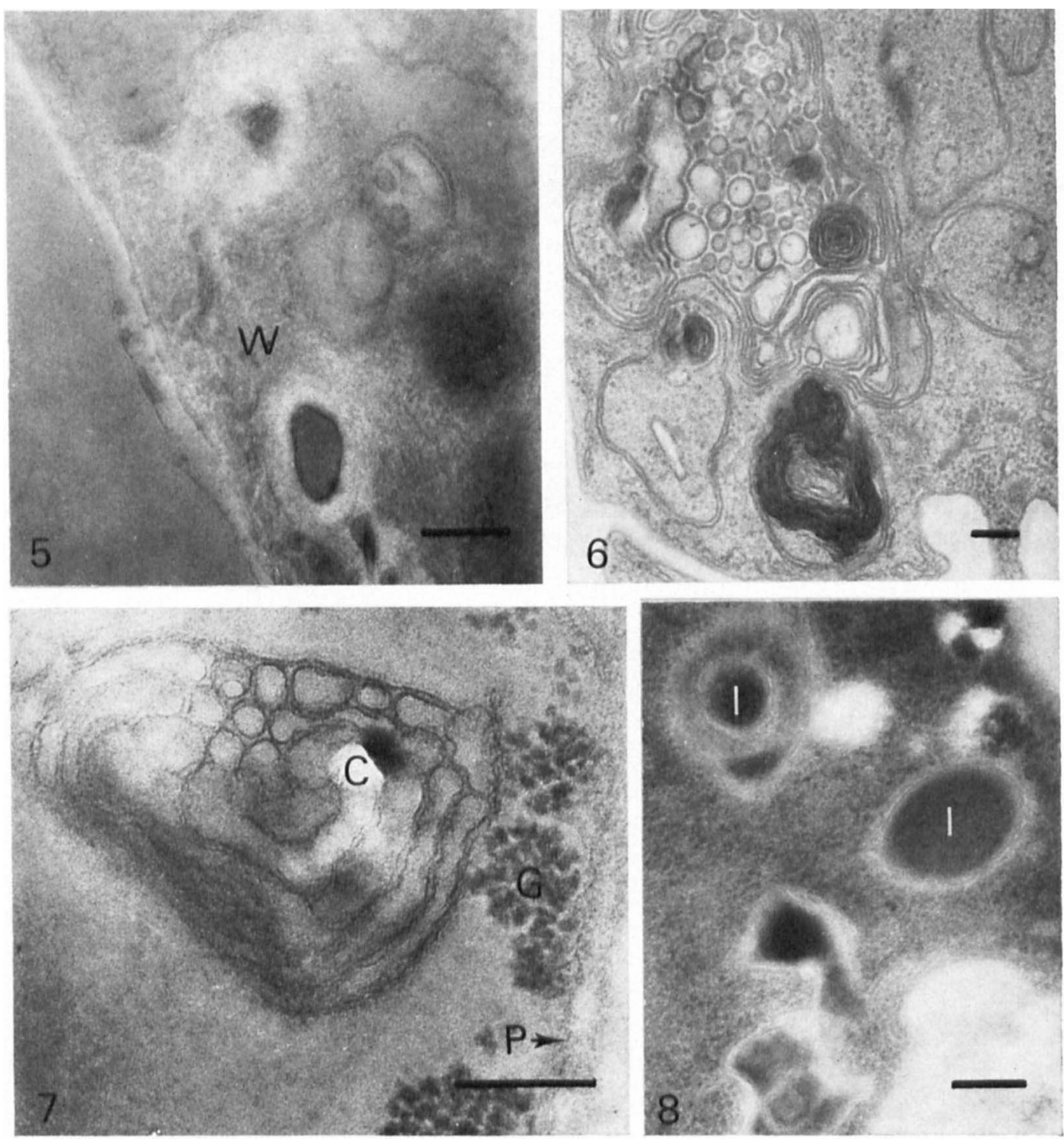

Bar markers represent $0.2 \mu \mathrm{m}$.

Fig. 5. Section of a hypha of the dome mutant stained by the Thiéry method. The large ingrowths (W) are contiguous with the hyphal wall.

Fig. 6. Complex membrane configurations in a hypha of the dome mutant.

Fig. 7. Complex membrane configuration (C), stained by the Thiéry method, in a hypha of the dome mutant. These membranes are heavily stained as are the adjacent glycogen granules (G). The plasmalemma $(P)$ is unstained.

Fig. 8. Hypha of the dome mutant extracted with sodium methoxide, showing electron-opaque material (I) remaining within wall ingrowths. 

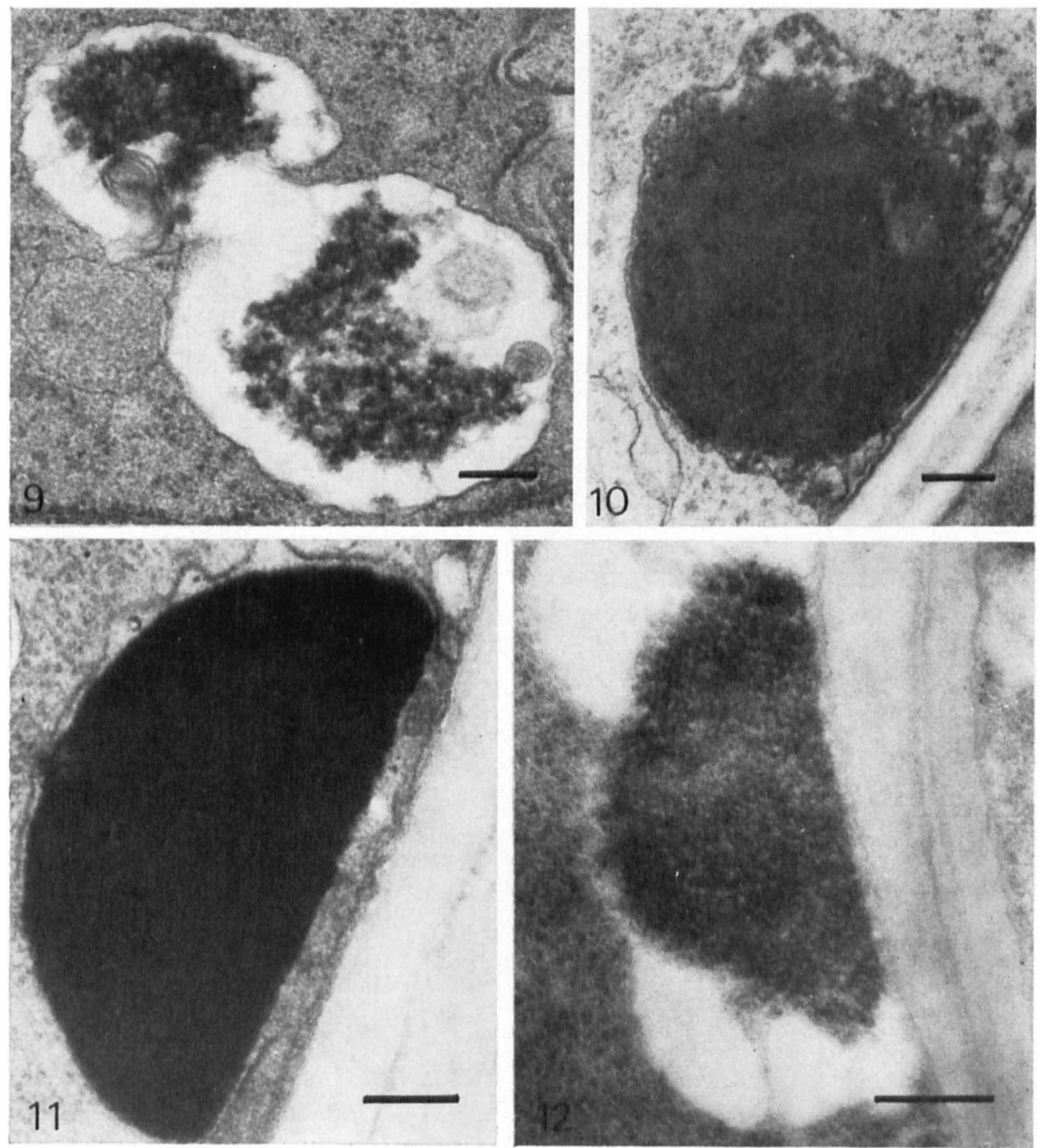

Bar markers represent $0.2 \mu \mathrm{m}$.

Figs 9 to I I. Stages in the condensation of electron-opaque material (indigotin) within vacuoles in hyphae of the dome mutant.

Fig. I2. Electron-opaque inclusion (indigotin) in a hypha of the dome mutant extracted with sodium methoxide. Note the complete removal of membranes, but not the pigment.

wall ingrowths in fungi is an unusual occurrence. Localized wall thickening has been reported in the yeast Rhodotorula glutinis grown in the presence of chloramphenicol (Smith \& Marchant, 1969) and this was associated with an inhibition of growth (Smith \& Marchant, 1968). An hypothesis relating to the dome mutant suggests that the wall ingrowths arise as a result of inhibition of hyphal extension coupled with localized excessive wall synthesis.

The electron-opaque material in the cytoplasm of the mutant strain appears in a number 
of forms each enclosed within a membrane. The different forms appear to represent stages in the condensation of material within a vacuole (Figs 9 to II). These structures are often adjacent to the wall, but there is no evidence for the passage of any material through it. The identity of the material within these structures is difficult to establish unequivocally, but it is osmiophilic and is not removed by sodium methoxide, whereas all the membranes of the cell are removed (Fig. I2). This observation seems to eliminate the possibility that it is lipid and, since structures of this type are absent in non-indigotin-producing strains, the most likely candidate appears to be the pigment indigotin. The opaque material within the wall ingrowths is similarly not removed with sodium methoxide (Fig. 8) and is also probably indigotin.

Indigotin is excreted into the medium by this strain, but there is no indication that granular indigotin is passed out of the cell. This leaves the conclusion that non-granular indigotin must pass out of the hyphae into the medium where it then forms particles, whereas indigotin which condenses in the hyphae remains permanently trapped. Support for this idea derives from the observation that dead hyphae frequently contain many indigotin granules.

Thanks are due to Mr S. F. Lowry, Experimental Officer in the University of Ulster Ultrastructure Unit, for his invaluable contributions to the completion of this work.

\section{REFERENCES}

EPSTEIN, E. \& MILES, P. G. (1966). Identification of indirubin as a pigment produced by mutant cultures of the fungus Schizophyllum commune. Botanical Magazine, Tokyo 79, 566-571.

EPSTEIN, E. \& MiLES, P. G. (1967). Identification of indole-3-acetic acid in the basidiomycete Schizophyllum commune. Plant Physiology, Lancaster 42, 9I I-9I4.

EURENIUS, L. \& JARSKÄR, R. (I970). A simple method to demonstrate lipids in epon-embedded ultrathin sections. Stain Technology 45, 129-132.

Koltin, Y. \& FleXer, A. S. (1969). Alteration of nuclear distribution in B-mutant strains of Schizophyllum commune. Journal of Cell Science 4, 739-749.

MARChANT, R. \& MOORE, R. T. (I973). Lomasomes and plasmalemmasomes in fungi. Protoplasma 76, 235-247.

Miles, P. G., Lund, H. \& Raper, J. R. (1956). The identification of indigo as a pigment produced by a mutant culture of Schizophyllum commune. Archives of Biochemistry and Biophysics 62, $1-5$.

Papazian, H. P. (1950). Physiology of the incompatibility factors in Schizophyllum commune. Botanical Gazette II2, I43-163.

RAPER, J. R. \& MILES, P. G. (1958). The genetics of Schizophyllum commune. Genetics, Princeton 43, $530-546$.

Raper, J. R., BAXter, M. G. \& Ellingboe, A. H. (I960). The genetic structure of the incompatibility factors of Schizophyllum commune. Proceedings of the National Academy of Sciences of the United States of America 46, 833-842.

RAUDASKoski, M. (I973). Light and electron microscope study of unilateral mating between a secondary mutant and a wild-type strain of Schizophyllum commune. Protoplasma 76, 35-48.

Smith, D. G. \& Marchant, R. (I968). Chloramphenicol inhibition of Pythium ultimum and Rhodotorula glutinis. Archiv für Mikrobiologie 6o, 262-274.

SMITH, D. G. \& MARCHANT, R. (I969). Unbalanced cell-wall synthesis in chloramphenicol-grown Rhodotorula glutinis. Antonie van Leeuwenhoek 35, II3-I 19.

SNIDER, P. J. \& RAPER, J. R. (1958). Nuclear migration in the basidiomycete Schizophyllum commune. American Journal of Botany 45, 538-546.

SwaCk, N. S. \& Miles, P. G. (1960). Conditions affecting growth and indigotin production by strain 130 of Schizophyllum commune. Mycologia 52, 574-583.

THIÉRY, J. P. (I967). Mise en évidence des polysaccharides sur coupes fines en microscopie électronique. Journal de Microscopie 6, 987-10I8.

Wessels, J. G. H. \& MARChANT, R. (1974). Enzymic degradation of septa in hyphal wall preparations from a monokaryon and a dikaryon of Schizophyllum commune. Journal of General Microbiology 83, 358368.

Wessels, J. G. H. \& NiederPRUEM, D. J. (1967). Role of a cell-wall glucan-degrading enzyme in mating of Schizophyllum commune. Journal of Bacteriology 94, I594-1602. 